\title{
Osteopontin is a marker for cancer aggressiveness and patient survival
}

\author{
GF Weber ${ }^{*}, 1,2$, GS Lett ${ }^{3}$ and NC Haubein ${ }^{3}$ \\ 'University of Cincinnati Academic Health Center, James L. Winkle College of Pharmacy, 3225 Eden Avenue, Cincinnati, OH 45267-0004, USA; \\ ${ }^{2}$ MetaMol Theranostics, Cincinnati, OH, USA; ${ }^{3}$ The BioAnalytics Group, Jamesburg, NJ, USA
}

\begin{abstract}
BACKGROUND: Only a fraction of molecular cancer markers identified in the scientific literature have found clinical use. Specifically, few predictors of invasiveness are established in diagnostics. Meta-analysis is a valuable tool for biomarker validation. Here, we evaluate Osteopontin as a marker for tumor aggressiveness (grade, stage, early progression) and patient survival.

METHODS: Publications through 2008 with the keywords 'osteopontin AND cancer' were retrieved. Titles and abstracts were screened for studies presenting original data on human subjects. This left 228 publications for data extraction. We applied categorical data analysis for testing the relationship between Osteopontin and a clinical variable.

RESULTS: Osteopontin ranks correlated with lower overall and disease-free/relapse-free survival in all tumors combined, as well as in lung cancer, breast cancer, prostate cancer, head and neck cancer, and liver cancer. Further, Osteopontin levels correlated with tumor grade and stage for all tumors combined and for several individual tumor types. Osteopontin levels were significantly associated with the early progression of eight cancers, independent in one, and inversely correlated in two.

CONCLUSIONS: Osteopontin is significantly associated with survival in several forms of cancer. Osteopontin levels are also markers for stage, grade, and early tumor progression in multiple cancers, reflecting a common molecular underpinning for distinct clinical measures. Osteopontin has value as a clinical tumor progression marker.

British Journal of Cancer (2010) 1 03, 86 I-869. doi:I0.1038/sj.bjc.6605834 www.bjcancer.com
\end{abstract}

(C) 2010 Cancer Research UK

Keywords: metastasis; survival; grade; stage

In recent years, substantial progress has been made in the detection and diagnosis of early stage cancers. This is mostly due to improved imaging technologies and new biomarkers in histological and hematologic testing. However, there still is a dearth of molecular indicators that distinguish highly aggressive tumors from moderately aggressive and non-aggressive ones. Specifically, few markers that predict invasiveness have been firmly established. Better molecular prognostics are needed to accurately assess disease. One candidate marker for the progression of various malignant tumors has been Osteopontin. In cancer, this molecule can support cell invasion and anchorage independence, thus enhancing tumor progression and metastasis formation (Weber, 2008). Despite a large literature on Osteopontin as a cancer marker, it is not in routine diagnostic use. One reason may be the diversity of source materials and cancer-associated readouts that have been investigated in correlation to Osteopontin levels. Therefore, it is important to analyze the comprehensive published evidence to discern which aspects of cancer pathophysiology are consistently associated with elevated Osteopontin levels, thus validating this molecule as a candidate marker.

The scientific literature on biomarkers has grown disproportionately more rapidly than the application of promising markers in clinical practice. Among the reasons for the delay are high barriers

*Correspondence: Dr GF Weber; E-mail: georg.weber@uc.edu Received II May 20I0; revised 5 July 20 I0; accepted 7 July 2010 in the regulatory process and limited available resources for the recruitment and analysis of sufficiently large patient populations. Meta-analysis is a suitable approach to enhancing knowledge about the diagnostic potential of individual biomarkers within these confines. Yet, conventional regression algorithms have had limited capability of combining distinct data sets and have therefore often fallen short of improving confidence. This is a particular problem for immunohistochemistry, where variable staining protocols combined with the semi-quantitative nature of the examination generate substantial study-to-study fluctuations. Categorical data analysis can limit such heterogeneity. The evaluation of within-study ranks results in a self-normalization of variable data sets. When applied to the meta-analysis of biomarkers, categorical data analysis has a dramatically higher sensitivity than conventional regression algorithms for detecting trends in data sets from disparate sources.

\section{METHODS}

\section{Data extraction}

A PubMed search with the keywords 'osteopontin AND cancer' through December 2008 resulted in 800 hits. Titles and abstracts were screened for studies involving human subjects, yielding 271 papers for initial analysis. 36 articles (including reviews, commentaries, experiments only on cell lines, no results on cancer, etc.) did not contain new data on Osteopontin in human cancer. 
Four articles were not obtained, even after request through interlibrary loan. Three papers were excluded because they contained one retraction, one article that pooled diverse primary tumors without separating them by tumor type, and one paper that applied scientifically questionable methodology (bidigital O-ring test). This left 228 publications to be used for data extraction (Table 1). Of articles not written in English, only the abstracts (not the full texts) were drawn on for obtaining data. For data extraction, numbers from the article text were applied directly; data presented in the format of graphs were measured and converted to the relevant units. Data from Kaplan-Meier survival curves were digitized using the software DataThief.

The cancers covered by the original publications include: breast cancer (34), ovarian cancer (25), liver cancer (21), lung cancer (20), head and neck cancer (15), colorectal cancer (14), gastric cancer (14), prostate cancer (13), bone cancer (9), oral cancer (9), melanoma (9), pancreatic cancer (8), renal cancer (8), esophageal cancer (7), glioma (7), mesothelioma (7), thyroid cancer (7), endometrial cancer (6), myeloma (6), cervical cancer (4), gestational trophoblastic tumor (4), leukemia/lymphoma (3), granular cell tumor (2), non-melanoma skin cancer (2), ampullary cancer (2), bladder cancer (2), medulloblastoma (2), soft tissue tumors (2), teratoid tumor (2), adrenocortical cancer (1), neuroblastoma (1), pilomatricoma (1), renal pelvis cancer (1), von Hippel-Lindau disease (1). The numbers in parentheses indicate the number of publications for each type of cancer. Note that several papers contain data on more than one type of cancer and are counted here for each. Therefore the sum is larger than the 228 original publications used for the data extraction.

\section{Data analysis}

A significance level of $95 \%(P<0.05)$ was applied to all studies. The correlation between Osteopontin expression levels and the clinical variables of interest was examined with a categorical approach (using ranked values). Within a study, the clinical variables were ranked from low to high and then normalized by the number of examples in the study. Studies that combined a range of grades were assigned the mean grade. Also within a study, the Osteopontin scores were ranked from low to high. In the case of immunohistochemistry scores that reported graded results on a $0-3^{+}$scale, a composite score for the study was computed by weighting each score by the fraction of patients reported for that score. For studies using an expanded scoring system, the scores were grouped at low, medium, and high levels and treated in the same way as the $0-3^{+}$results. For studies that only reported mean or median results, the raw values were simply ranked. Ranking accomplishes a self-normalization within each study (Hong et al, 2006; Hong and Breitling, 2008) and permits the simultaneous analysis of both the summary results (mean, median only) and various graded results. In the case of immunohistochemistry, this reduces the effects of different pathologists scoring the samples. In other assay types, such as ELISA or quantitative RT-PCR, this eliminates the need for a normal standard under the assumption that all samples within a study are compared against the same standard.

We utilized the Pearson $\chi^{2}$ test (Agresti, 2007) for independence to assess whether the Osteopontin ranks are independent of the clinical variable ranks. This test was carried out by constructing contingency tables using the ranks for each variable, and populating each cell with the total number of patients reporting that combination of ranks. Separate tables were constructed for sets of studies with 2, 3, or more ranks to avoid structural zeros. The Mantel-Haenszel $\chi^{2}$ test (Agresti, 2007) was used to assess the hypothesis that the ranking of a particular clinical variable within a study is linearly related to the Osteopontin level. We then tested for a non-linear trend by examining the residuals between the observed values and a linear model of the data.
Receiver operator characteristic (ROC) curves are commonly used to assess diagnostic performance of a particular measurable quantity. The most common feature used to quantify this characteristic is the area under the curve, which can be interpreted here as the probability that for two randomly chosen samples, the one with the higher Osteopontin rank will also have a higher rank for the clinical variable in question (Rice and Harris, 2005). In the case of the ranked data in this study, that probability can be calculated for each clinical study. Each pair of patient groups in the study was examined, and the fraction of those where a group with higher clinical variable rank also had a higher Osteopontin level rank is reported here. The statistical significance of this fraction was tested by carrying out a Monte Carlo simulation to estimate the distribution of fractions expected for random ranks.

\section{Reporting standards}

The data applied to this study were not skewed by publication bias according to a funnel plot analysis. The present study has been conducted according to the standards of the PRISMA Statement (Moher et al, 2009).

\section{RESULTS}

\section{Osteopontin in patient survival}

We applied categorical meta-analysis to the evaluation of Osteopontin as a prognostic marker. The distribution of ranks for published overall and disease-free/relapse-free survival versus measured Osteopontin levels displayed an aggregation along the diagonal in bar graphs, indicating a good correspondence for higher Osteopontin rank to lower mean survival time (Figure $1 \mathrm{~A}$ and $\mathrm{B})$. To further quantify these results, we determined the probability that for two patient groups selected at random from a study, the one that had the higher Osteopontin score would also have a shorter mean survival time. This resulted in a probability of $90.8 \%, P<1 \times 10^{-5}$ for overall survival and a probability of $92.9 \%$, $P=1 \times 10^{-4}$ for disease-free/relapse-free survival, where the significance was estimated using a permutation test. These results indicate that Osteopontin rank is a good predictor of survival outcome rank within a study. The actual probability calculated from the meta-analysis of the data was significant when compared to the estimated probability distribution under the null hypothesis that Osteopontin and mean survival time are independent (Figure 1C and D). When broken down to individual cancers, the association between Osteopontin levels and overall survival was significant for lung cancer, breast cancer, prostate cancer, head and neck cancer, and liver cancer (Table 2). Similar results were obtained using the meta-analysis function in Oncomine (Supplement 1). For several cancer types, only one published study was available. Those cases were excluded from the meta-analysis.

In clinical practice, the detection of Osteopontin is particularly important in two settings. In serum or plasma, Osteopontin may serve as a prognostic marker associated with a minimally invasive procedure. After a biopsy, Osteopontin may serve as a prognostic marker directly linked to the tumor. Therefore, we separately analyzed the patient survival data for Osteopontin in these distinct types of specimens. For all cancers combined, the levels of Osteopontin in plasma, in serum, and in tumors significantly identified subpopulations with shorter mean survival (Table 3 ). In tumors, the highest Osteopontin groups had a mean survival 850 days shorter than the lowest Osteoponin groups. For plasma, the highest Osteopontin groups had a mean survival 560 days shorter than the lowest Osteoponin groups. The concordance between Osteopontin ranks and risk for reduced survival was confirmed for several individual cancers (Table 3). However, the sample sizes for several individual cancers were not sufficiently 
Table I Source references for data extraction

\begin{tabular}{|c|c|c|c|}
\hline I & Bachmann IM, Ladstein RG, Straume O, Naumov GN, Akslen LA. & $(2008)$ & BMC Cancer. 8, 362 \\
\hline 2 & Cappia S, Righi L, Mirabelli D, Ceppi P, Bacillo E, et al. & (2008) & Am J Clin Pathol. 130, 58 \\
\hline 3 & Carlos-Bregni R, Contreras E, Hiraki KR, Vargas PA, León JE, et al. & $(2008)$ & Oral Surg Oral Med Oral Pathol Oral Radiol Endod. 105, e47 \\
\hline 4 & Carrer A, Zacchigna S, Balani A, Pistan V, Adami A, et al. & (2008) & Eur J Cancer. 44, 1761 \\
\hline 5 & Caruso DJ, Carmack AJ, Lokeshwar VB, Duncan RC, Soloway MS, et al. & (2008) & Clin Cancer Res. 14, 4III \\
\hline 6 & Castellano G, Malaponte G, Mazzarino MC, Figini M, Marchese F, et al. & (2008) & Clin Cancer Res. 14, 7470 \\
\hline 7 & Chang PL, Harkins L, Hsieh YH, Hicks P, Sappayatosok K, et al. & (2008) & J Histochem Cytochem. 56, 57 \\
\hline 8 & Cho H, Hong SW, Oh YJ, Kim MA, Kang ES, et al. & (2008) & J Cancer Res Clin Oncol. 134, 909 \\
\hline 9 & Creaney J, Yeoman D, Demelker Y, Segal A, Musk AW, et al. & (2008) & J Thorac Oncol. 3, 85I \\
\hline 10 & Fredriksson S, Horecka J, Brustugun OT, Schlingemann J, Koong AC, et al. & (2008) & Clin Chem. 54, 582 \\
\hline 11 & Galamb $\mathrm{O}$. & (2008) & On Hetil. 149, 1373. Hungarian. \\
\hline 12 & Hui EP, Sung FL, Yu BK, Wong CS, Ma BB, et al. & $(2008)$ & Clin Cancer Res. 14, $7080^{\circ}$ \\
\hline 13 & Katase N, Tamamura R, Gunduz M, Murakami J, Asaumi J, et al. & (2008) & Head Face Med. 4, 28 \\
\hline 14 & Kato N, Motoyama T. & (2008) & Histopathology. 52, 682 \\
\hline 15 & Kittaka N, Takemasa I, Takeda Y, Marubashi S, Nagano H, et al. & (2008) & Eur J Cancer. 44, 885 \\
\hline 16 & Korita PV, Wakai T, Shirai Y, Matsuda Y, Sakata J, et al. & (2008) & Hum Pathol. 39, 1777 \\
\hline 17 & Lee CY, Tien HF, Hou HA, Chou WC, Lin LI. & (2008) & BrJ Haematol.141, 736 \\
\hline 18 & Mack PC, Redman MW, Chansky K, Williamson SK, Farneth NC, et al. & (2008) & J Clin Oncol. 26, 477| \\
\hline 19 & Matusan-llijas K, Behrem S, Jonjic N, Zarkovic K, Lucin K. & (2008) & Pathol Oncol Res. 14, 293 \\
\hline 20 & McAllister SS, Gifford AM, Greiner AL, Kelleher SP, Saelzler MP, et al. & (2008) & Cell. 133, 994 \\
\hline 21 & Mirza M, Shaughnessy E, Hurley JK, Vanpatten KA, Pestano GA, et al. & (2008) & Int J Cancer. 122, 889 \\
\hline 22 & Moore RG, Brown AK, Miller MC, Skates S, Allard WJ, et al. & (2008) & Gynecol Oncol. 108, 402 \\
\hline 23 & Mrochem J, Sodowski K, Deja R, Walaszek-Gruszka A, Wojcieszek A, et al. & (2008) & Ginekol Pol. 79, 27I. Polish \\
\hline 24 & Ohike N, Sato M, Kawahara M, Ohyama S, Morohoshi T. & (2008) & JOP. 9, 335 \\
\hline 25 & Oler G, Camacho CP, Hojaij FC, Michaluart P Jr, Riggins GJ, et al. & $(2008)$ & Clin Cancer Res. 14, 4735 \\
\hline 26 & Patani N, Jiang W, Mokbel K. & (2008) & Int J Cancer. 122, 2646 \\
\hline 27 & Rangel J, Nosrati M, Torabian S, Shaikh L, Leong SP, et al. & (2008) & Cancer. 112,144 \\
\hline 28 & Ribeiro-Silva A, Oliveira da Costa JP. & $(2008)$ & Int J Biol Markers. 23, 154 \\
\hline 29 & Shimizu S, Tsukada J, Sugimoto T, Kikkawa N, Sasaki K, et al. & (2008) & Int J Cancer. 123, 1816 \\
\hline 30 & Tang H, Wang J, Bai F, Zhai H, Gao J, et al. & (2008) & Cancer Invest. 26, 60 \\
\hline 31 & Tun HW, Personett D, Baskerville KA, Menke DM, Jaeckle KA, et al. & (2008) & Blood. III, 3200 \\
\hline 32 & Vergis R, Corbishley CM, Norman AR, Bartlett J, Jhavar S, et al. & (2008) & Lancet Oncol. 9, 342 \\
\hline 33 & Visintin I, Feng Z, Longton G, Ward DC, Alvero AB, et al. & (2008) & Clin Cancer Res. 14, 1065 \\
\hline 34 & Wang X, Chao L, Ma G, Chen L, Tian B, et al. & (2008) & Eur J Clin Invest. 38, 438 \\
\hline 35 & Wu IC, Wu MT, Chou SH, Yang SF, Goan YG, et al. & $(2008)$ & World J Surg. 32, 1989 \\
\hline 36 & Yang GH, Fan J, Xu Y, Qiu SJ, Yang XR, et al. & (2008) & Oncologist. 13, 1155 \\
\hline 37 & Zdzisinska B, Bojarska-Junak A, Dmoszynska A, Kandefer-Szerszen M. & (2008) & Arch Immunol Ther Exp (Warsz). 56, 207 \\
\hline 38 & Zhao J, Lu B, Xu H, Tong X, Wu G, et al. & (2008) & Hepatology. 48, 265 \\
\hline 39 & Zhao L, Li T, Wang Y, Pan Y, Ning H, et al. & (2008) & Int J Clin Pract. 62, 1056 \\
\hline 40 & Alonso SR, Tracey L, Ortiz P, Pérez-Gómez B, Palacios J, et al. & (2007) & Cancer Res. 67, 3450 \\
\hline 41 & Bao LH, Sakaguchi H, Fujimoto J, Tamaya T. & (2007) & Jiomed Sci. 14, 373 \\
\hline 42 & Bloomston M, Ellison EC, Muscarella P, Al-Saif O, Martin EW, et al. & (2007) & Ann Surg Oncol. 14, 211 \\
\hline 43 & Chandran UR, Ma C, Dhir R, Bisceglia M, Lyons-Weiler M, et al. & (2007) & BMC Cancer. 7, 64 \\
\hline 44 & Chang YS, Kim HJ, Chang J, Ahn CM, Kim SK, et al. & (2007) & Lung Cancer. 57, 373 \\
\hline 45 & Dai N, Bao Q, Lu A, Li J. & (2007) & Oncology. 72, 89 \\
\hline 46 & Del Sordo R, Cavaliere A, Sidoni A. & $(2007)$ & Am J Dermatopathol. 29, 470 \\
\hline 47 & Dizdar O, Barista I, Kalyoncu U, Karadag $\mathrm{O}$, Hascelik G, et al. & $(2007)$ & Am J Hematol. 82, 185 \\
\hline 48 & Eto M, Kodama S, Nomi N, Uemura N, Suzuki M. & (2007) & Auris Nasus Larynx. 34, 343 \\
\hline 49 & Frey AB, Wali A, Pass H, Lonardo F. & (2007) & Histopathology. 50, 720 \\
\hline 50 & Gallot D, Marceau G, Laurichesse-Delmas H, Vanlieferinghen P, Dechelotte PJ, et al. & (2007) & Fetal Diagn Ther. 22, 161 \\
\hline 51 & Ghert M, Simunovic N, Cowan RW, Colterjohn N, Singh G. & $(2007)$ & Clin Orthop Relat Res. 459, 8 \\
\hline 52 & Grigoriu BD, Scherpereel A, Devos P, Chahine B, Letourneux M, et al. & $(2007)$ & Clin Cancer Res. 13, 2928 \\
\hline 53 & Grisaru D, Hauspy J, Prasad M, Albert M, Murphy KJ, et al. & (2007) & Oncol Rep. 18, 1347 \\
\hline 54 & Guglielmi G, Ciberti A, Foddis R, Ambrosino N, Chella A, et al. & (2007) & G Ital Med Lav Ergon. 29, 345. Italian \\
\hline 55 & Gui SY, Li HH, Zuo L, Zhou Q, Wu Q, et al. & (2007) & Zhonghua Yi Xue Za Zhi. 87, 3219. Chinese \\
\hline 56 & Higashiyama M, Ito T, Tanaka E, Shimada Y. & (2007) & Ann Surg Oncol. 14, 3419 \\
\hline 57 & Hu Z, Xiao T, Lin DM, Guo SP, Zhang ZQ, et al. & $(2007)$ & Zhonghua Zhong Liu Za Zhi. 29, 59I. Chinese \\
\hline 58 & Jaeger J, Koczan D, Thiesen HJ, Ibrahim SM, Gross G, et al. & (2007) & Clin Cancer Res. 13, 806 \\
\hline 59 & Katakura A, Kamiyama I, Takano N, Shibahara T, Muramatsu T, et al. & (2007) & Bull Tokyo Dent Coll. 48, 199 \\
\hline 60 & Kuroda N, Hamaguchi N, Ohara M, Hirouchi T, Mizuno K, et al. & (2007) & Med Mol Morphol. 40, 218 \\
\hline 61 & Le QT, Kong C, Lavori PW, O'byrne K, Erler JT, et al. & (2007) & Int J Radiat Oncol Biol Phys. 69, 167 \\
\hline 62 & Lee YC, Pan HW, Peng SY, Lai PL, Kuo WS, et al. & $(2007)$ & Eur J Cancer. 43, 736 \\
\hline 63 & Li Y, Lu Y, Ceng Y, Yang X. & (2007) & $\begin{array}{l}\text { Lin Chung Er Bi Yan Hou Tou Jing Wai Ke Za Zhi. } \\
\text { 21, 121. Chinese }\end{array}$ \\
\hline 64 & Lin HM, Chatterjee A, Lin YH, Anjomshoaa A, Fukuzawa R, et al. & $(2007)$ & Oncol Rep. 17, 1541 \\
\hline 65 & Matsuzaki H, Shima K, Muramatsu T, Ro Y, Hashimoto S, et al. & (2007) & J Oral Pathol Med. 36, 30 \\
\hline 66 & Meinhold-Heerlein I, Bauerschlag D, Zhou Y, Sapinoso LM, Ching K, et al. & (2007) & Clin Cancer Res. 13, 458 \\
\hline 67 & Mountzios G, Dimopoulos MA, Bamias A, Papadopoulos G, Kastritis E, et al. & (2007) & Acta Oncol. 46, 221 \\
\hline 68 & Mountzios G, Dimopoulos MA, Bamias A, Papadopoulos G, Kastritis E, et al. & (2007) & Acta Oncol. 46, 221 \\
\hline 69 & Nordsmark M, Eriksen JG, Gebski V, Alsner J, Horsman MR, et al. & (2007) & Radiother Oncol. 83, 389 \\
\hline 70 & Ogbureke KU, Nikitakis NG, Warburton G, Ord RA, Sauk J, et al. & (2007) & Oral Oncol. 43, 920 \\
\hline 71 & Pascaretti-Grizon F, Gaudin-Audrain C, Gallois Y, Retaillaud-Gaborit N, Baslé MF. & (2007) & Morphologie. 91, 180 \\
\hline 72 & Ramankulov A, Lein M, Kristiansen G, Loening SA, Jung K. & (2007) & Prostate. 67, 330 \\
\hline
\end{tabular}


Table I (Continued)

73 Ramankulov A, Lein M, Kristiansen G, Meyer HA, Loening SA et al.

74 Reiniger IW, Wolf A, Welge-Lüssen U, Mueller AJ, Kampik A, et al. Robbiani DF, Colon K, Ely S, Ely S, Chesi M, et al.

Rohde F, Rimkus C, Friederichs J, Rosenberg R, Marthen C, et al.

Said HM, Hagemann C, Staab A, Stojic J, Kühnel S, et al.

Sakaguchi $H$, Fujimoto J, Hong BL, Tamaya T.

Shin HD, Park BL, Cheong HS, Yoon $H$, Kim YJ, et al.

Soikkeli J, Lukk M, Nummela P, Virolainen S, Jahkola T, et al.

Staibano S, Merolla F, Testa D, lovine R, Mascolo M, et al.

Tigrani DY, Weydert JA.

Winfield HL, Kirkland F, Ramos-Ceballos FI, Horn TD.

Wu CY, Wu MS, Chiang EP, Wu CC, Chen Y], et al.

Xie $\mathrm{H}$, Song J, Du R, Liu K, Wang J, et al.

Allan AL, George R, Vantyghem SA, Lee MW, Hodgson NC, et al. Bache M, Reddemann R, Said HM, Holzhausen HJ, Taubert H, et al. Benoist-Lasselin C, de Margerie E, Gibbs L, Cormier S, Silve C, et al. Bramwell VH, Doig GS, Tuck AB, Wilson SM, Tonkin KS, et al. Briese J, Schulte HM, Bamberger CM, Löning T, Bamberger AM.

Colin C, Baeza N, Bartoli C, Fina F, Eudes N, et al.

Dalla-Torre CA, Yoshimoto M, Lee CH, Joshua AM, de Toledo SR, et al.

Darling MR, Gauthier M, Jackson-Boeters L, Daley TD, Chambers AF, et al.

Debucquoy A, Goethals L, Geboes K, Roels S, Mc Bride WH, et al.

Feng HC, Tsao SW, Ngan HY, Kwan HS, Shih SM, et al.

Fluge $\varnothing$, Bruland $O$, Akslen LA, Lillehaug JR, Varhaug JE.

Forootan SS, Foster CS, Aachi VR, Adamson J, Smith PH, et al.

Gong YH, Sun LP, Yuan Y.

Hashiguchi $Y$, Tsuda $H$, Bandera CA, Nishimura S, Inoue T, et al.

Huang J, Sheng HH, Shen T, Hu YJ, Xiao HS, et al.

Kadkol SS, Lin AY, Barak V, Kalickman I, Leach L, et al.

Kim J, Ki SS, Lee SD, Han C], Kim YC, et al.

Kita Y, Natsugoe S, Okumura H, Matsumoto M, Uchikado Y, et al.

Köbel M, Langhammer T, Hüttelmaier S, Schmitt WD, Kriese K, et al.

Koopmann J, Rosenzweig CN, Zhang Z, Canto MI, Brown DA, et al.

Le OT, Chen E, Salim A, Cao H, Kong CS, et al.

Luo JH, Ren B, Keryanov S, Tseng GC, Rao UN, et al.

Matusan K, Dordevic G, Stipic D, Mozetic V, Lucin K.

Miller CT, Lin L, Casper AM, Lim J, Thomas DG, et al.

Nakae M, Iwamoto I, Fujino T, Maehata Y, Togami S, et al.

Petrik D, Lavori PW, Cao H, Zhu Y, Wong P, et al.

Rogers CD, Fukushima N, Sato N, Shi C, Prasad N, et al.

Rudland S, Martin L, Roshanlall C, Winstanley J, Leinster S, et al.

Sando N, Oka K, Moriya T, Saito H, Nagakura S, et al.

Vordermark D, Said HM, Katzer A, Kuhnt T, Hänsgen G, et al.

Wang G, Platt-Higgins A, Carroll J, de Silva Rudland S, Winstanley J, et al.

Wong YF, Cheung TH, Tsao GS, Lo KW, Yim SF, et al.

Ye B, Skates S, Mok SC, Horick NK, Rosenberg HF, et al.

Yuan RH, Jeng YM, Chen HL, Lai PL, Pan HW, et al.

Zhang $\mathrm{H}$, Ye QH, Ren N, Zhao L, Wang YF, et al.

Zhang HZ, Liu JG, Wei YP, Wu C, Cao YK, et al.

Ang C, Chambers AF, Tuck AB, Winquist E, lzawa Jl.

Boldrini L, Donati V, Dell'Omodarme M, Prati MC, Faviana P, et al.

Bramwell VH, Tuck AB, Wilson SM, Stitt LW, Cherian AK, et al.

Briese J, Oberndörfer M, Schulte HM, Löning T, Bamberger AM.

Celetti A, Testa D, Staibano S, Merolla F, Guarino V, et al.

Donati V, Boldrini L, Dell'Omodarme M, Prati MC, Faviana P, et al.

Guarino V, Faviana P, Salvatore G, Castellone MD, Cirafici AM, et al.

Hoshi N, Sugino T, Suzuki T.

Hu Z, Lin D, Yuan J, Xiao T, Zhang $H$, et al.

Iso Y, Sawada T, Okada T, Kubota K

Kao CL, Chiou SH, Chen YJ, Singh S, Lin HT, et al.

Kao CL, Chiou SH, Ho DM, Chen YJ, Liu RS, et al.

Kawakami T, Shimizu T, Kimura A, Hasegawa $\mathrm{H}$, Siar $\mathrm{CH}$, et al.

Libra M, Indelicato M, De Re V, Zignego AL, Chiocchetti A, et al.

Matusan K, Dordevic G, Mozetic V, Lucin K.

Mor G, Visintin I, Lai Y, Zhao H, Schwartz P, et al.

O'Neill C, Deavers MT, Malpica A, Foster H, McCluggage WG.

Overgaard J, Eriksen JG, Nordsmark M, Alsner J, Horsman MR, et al.

Pass HI, Lott D, Lonardo F, Harbut M, Liu Z, et al.

Peng SY, Ou YH, Chen WJ, Li HY, Liu SH, et al.

Rosen DG, Wang L, Atkinson JN, Yu Y, Lu KH, et al.

Sedivy R, Kalipciyan M, Mazal PR, Wolf B, Wrba F, et al.

Sedivy R, Peters K, Klöppel G.

Shimada Y, Watanabe G, Kawamura J, Soma T, Okabe M, et al.
(2007)

(2007)

(2007)

(2007)

(2007)

(2007)

(2007)

(2007)

(2007)

(2007)

(2007)

(2007)

(2007)

(2006)

(2006)

(2006)

(2006)

(2006)

(2006)

(2006)

(2006)

(2006)

(2006)

(2006)

(2006)

(2006)

(2006)

(2006)

(2006)

(2006)

(2006)

(2006)

(2006)

(2006)

(2006)

(2006)

(2006)

(2006)

(2006)

(2006)

(2006)

(2006)

(2006)

(2006)

(2006)

(2006)

(2006)

(2006)

(2006)

(2005)

(2005)

(2005)

(2005)

(2005)

(2005)

(2005)

(2005)

(2005)

(2005)

(2005)

(2005)

(2005)

(2005)

(2005)

(2005)

(2005)

(2005)

(2005)

(2005)

(2005)

(2005)

(2005)

(2005)
J Cancer Res Clin Oncol. 133, 643

Am J Ophthalmol. 143, 705

Hematol Oncol. 25, 16

Int J Cancer. 121, 1717

Radiother Oncol. 83, 398

Cancer Lett. 247, 98

Int J Epidemiol. 36, 1001

J Pathol. 213, 180

Br J Cancer. 97, 1545

Am J Clin Pathol. 127, 580

Arch Dermatol. 143, 1076

Gut. 56, 782

Dig Liver Dis. 39, 167

Am J Pathol. 169, 233

Int J Radiat Oncol Biol Phys. 66, I48I

Bone. 39, 17

Clin Cancer Res. 12, 3337

Int J Gynecol Pathol. 25, 161

Oncogene. 25, 2818

BMC Cancer. 6, 237

Oral Oncol. 42, 363

Radiother Oncol. 80, 172

Placenta. 27, 521

Thyroid. 16, 161

Int | Cancer. I| 8, 2255

Zhonghua Zhong Liu Za Zhi. 28, 69l. Chinese

Med Oncol. 23, 205

FEBS Lett. 580, 357

Invest Ophthalmol Vis Sci. 47, 802

Am J Gastroenterol. I01, 205 I

Br J Cancer. 95, 634

Mod Pathol. 19, 581

Clin Cancer Res. 12, 442

Clin Cancer Res. 12, 1507

Hepatology. 44, 1012

J Surg Oncol. 94, 325

Oncogene. 25, 409

J Obstet Gynaecol Res. 32, 309

| Clin Oncol. 24, 529|

Cancer Biol Ther. 5, 1383

Clin Cancer Res. 12, 1192

APMIS. I |4, 58|

BMC Cancer. 6, 207

Cancer Res. 66, 1199

Int J Cancer. | | 8, 246 |

Clin Cancer Res. 12, 432

J Pathol. 209, 549

J Cancer Res Clin Oncol. 132, 709

Nan Fang Yi Ke Da Xue Xue Bao. 26, 1612. Chinese

BJU Int. 96, 803

$\mathrm{Br} J$ Cancer. 93, 453

Cancer Biol Ther. 4, 1336

Int J Gynecol Pathol. 24, 27 I

Clin Cancer Res. 11, 8019

Clin Cancer Res. II, 6459

J Clin Endocrinol Metab. 90, 5270

Pathol Int. 55, 484

Clin Cancer Res. II, 4646

J Surg Oncol. 92, 304

Mod Pathol. 18, 769

Am I Clin Pathol. 123, 297

Eur J Med Res. 10, 475

Cancer Biol Ther. 4, 1192

Pathol Oncol Res. II, 108

Proc Natl Acad Sci USA. 102, 7677

Am J Surg Pathol. 29, 1034

Lancet Oncol. 6, 757

N Engl J Med. 353, 1564

Int J Oncol. 26, 1053

Gynecol Oncol. 99, 267

Cancer Detect Prev. 29, 8

Virchows Arch. 446, 41

Oncology. 68, 285 
Table I (Continued)

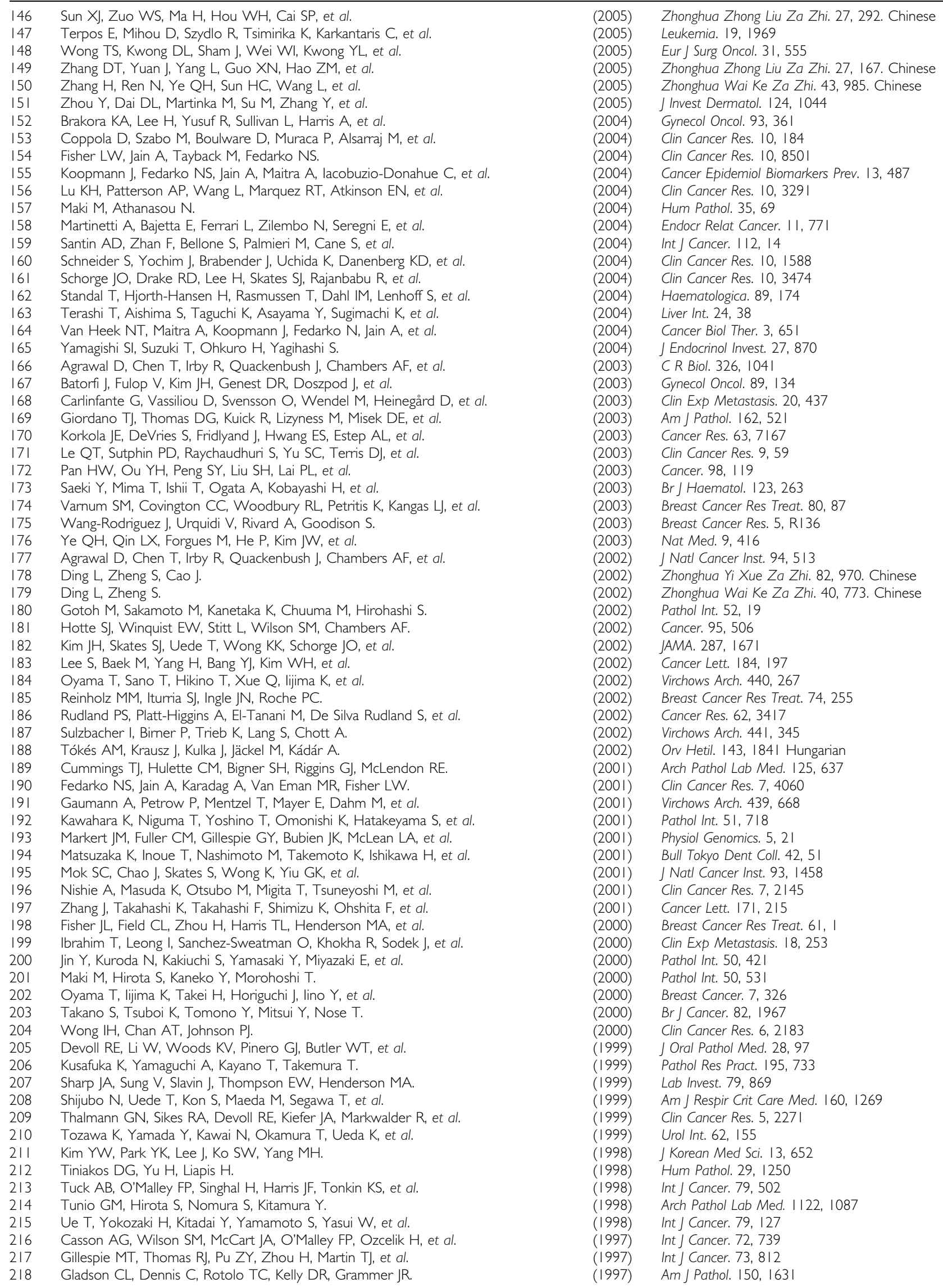


Table I (Continued)

\begin{tabular}{|c|c|c|c|}
\hline 219 & Singhal $\mathrm{H}$, Bautista DS, Tonkin KS, O'Malley FP, Tuck AB, et al. & (1997) & Clin Cancer Res. 3, 605 \\
\hline 220 & Tuck AB, O'Malley FP, Singhal H, Tonkin KS, Harris JF, et al. & $(1997)$ & Arch Pathol Lab Med. 121, 578 \\
\hline 221 & Bautista DS, Denstedt J, Chambers AF, Harris JF. & $(1996)$ & J Cell Biochem. 61, 402 \\
\hline 222 & Chambers AF, Wilson SM, Kerkvliet N, O'Malley FP, Harris JF, et al. & $(1996)$ & Lung Cancer. 15, 311 \\
\hline 223 & Bellahcène $A$, Castronovo V. & $(1995)$ & Am J Pathol. 146, 95 \\
\hline 224 & Hirota S, Asada H, Kohri K, Tsukamoto Y, Ito A, et al. & $(1995)$ & J Invest Dermatol. 105, 138 \\
\hline 225 & Hirota S, Ito A, Nagoshi J, Takeda M, Kurata A, et al. & $(1995)$ & Lab Invest. 72, 64 \\
\hline 226 & Saitoh Y, Kuratsu J, Takeshima H, Yamamoto S, Ushio Y. & $(1995)$ & Lab Invest. 72, 55 \\
\hline 227 & Brown LF, Papadopoulos-Sergiou A, Berse B, Manseau EJ, Tognazzi K, et al. & $(1994)$ & Am J Pathol. 145, 610 \\
\hline 228 & Senger DR, Perruzzi CA, Papadopoulos A. & $(1989)$ & Anticancer Res. 9, 1291 \\
\hline
\end{tabular}

PubMed references for 'osteopontin AND cancer' were screened for studies involving human subjects and then filtered for the presentation of original data. This left 228 publications to be used for data extraction. The references are listed in reverse chronologic order. For space considerations, titles are omitted. Foreign language articles are indicated as such.

A

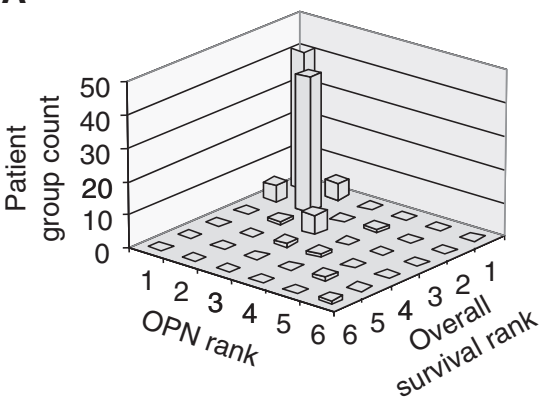

B

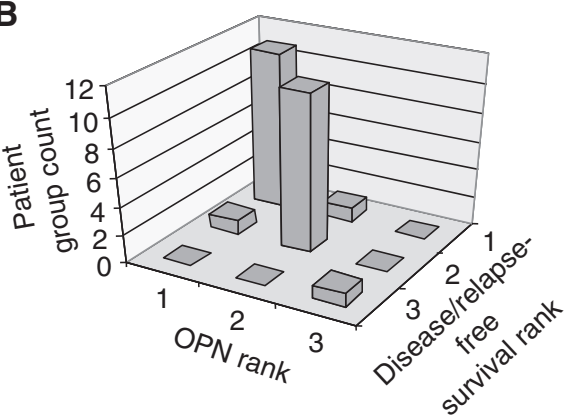

C

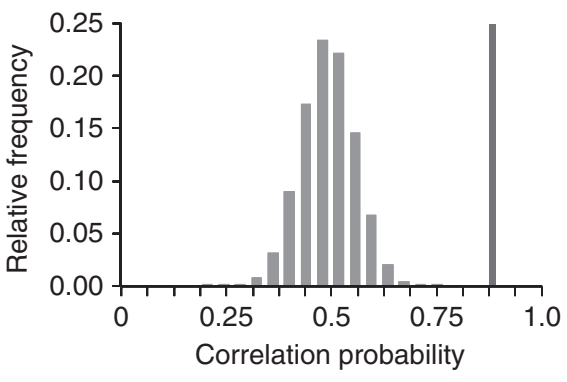

D

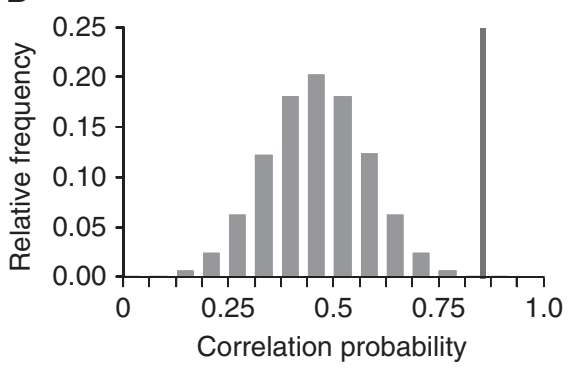

Figure I Osteopontin in overall survival and in disease-free/relapse-free survival. (A) Overall survival and Osteopontin ranks for all cancers combined. (B) Disease-free survival and Osteopontin ranks for all cancers combined. (C) Probability Distribution Function for independent Osteopontin and overall survival ranks. The measured value for Osteopontin data is shown as a vertical line. (D) Probability Density Function for independent Osteopontin and disease free survival ranks. Measured value for Osteopontin data is shown as a vertical line.

Table 2 Osteopontin and survival in individual cancers

\begin{tabular}{lccc}
\hline Cancer & Concordance & P-value & Studies \\
\hline Lung & 1.000 & $\mathbf{0 . 0 0 1}$ & 3 \\
Breast & 0.917 & $\mathbf{0 . 0 0 4}$ & 8 \\
Prostate & 1.000 & $\mathbf{0 . 0 1 3}$ & 3 \\
Head and neck & 1.000 & $\mathbf{0 . 0 2 0}$ & 4 \\
Liver & 0.875 & $\mathbf{0 . 0 3 3}$ & 8 \\
Cervical & 1.000 & 0.126 & 3 \\
Esophageal & 1.000 & 0.126 & 3 \\
Gastric & 0.750 & 0.189 & 3 \\
Kidney & 1.000 & 0.249 & 2 \\
Mesothelioma & 1.000 & 0.249 & 2 \\
\hline
\end{tabular}

Published curves for overall survival were digitised for analysis. P-values in bold are considered significant. They indicate that Osteopontin over-expression is associated with elevated risk for death from cancer. For several cancer types, only one published study was available. Those cases were excluded from the meta-analysis. Shown are only cancers for which more than one published study was available for evaluation. large to obtain $95 \%$ significance for the rank statistic used here (in plasma for gastric, cervical, liver, teratoid, esophageal, and renal cancers; in serum for breast cancer, head and neck cancer, and mesothelioma; in tumors for colorectal, ovarian, and prostate cancers, mesothelioma, and glioma). In tumors, discordance (i.e. higher Osteopontin groups had longer mean survival times) was observed for one study each on bone cancer, endometrial cancer, and melanoma.

\section{Osteopontin in tumor grade, stage and early progression}

Osteopontin immunohistochemistry score ranks and tumor grade and stage ranks were dependent $(P<0.001)$ for all cancers combined (Figure 2A), as well as for 12 individual cancers for grade, 13 individual cancers for stage T, 8 individual cancers for stage $\mathrm{N}$, and 9 individual cancers for stage $\mathrm{M}$ (Table 4). Graphical representation of the group ranks suggested a strong positive relationship, reflected in a high density of data points along the 
Table 3 Osteopontin and survival in distinct clinical specimens

\begin{tabular}{|c|c|c|c|}
\hline & Number of studies & Concordance & $P$-value \\
\hline \multicolumn{4}{|l|}{ Specimen } \\
\hline \multicolumn{4}{|c|}{ (A) All tumors combined } \\
\hline Tumor & 31 & 0.825 & $<\mathbf{0 . 0 0 0 1}$ \\
\hline Plasma & 14 & । & $<\mathbf{0 . 0 0 0 1}$ \\
\hline Serum & 3 & I & 0.04 \\
\hline \multicolumn{4}{|l|}{ Cancer type } \\
\hline \multicolumn{4}{|c|}{ (B) Plasma, individual cancers } \\
\hline Lung & । & I & 0.00 \\
\hline Prostate & 2 & 1 & 0.08 \\
\hline Breast & 2 & I & 0.08 \\
\hline Head and neck & 3 & i & 0.13 \\
\hline \multicolumn{4}{|l|}{ Cancer type } \\
\hline \multicolumn{4}{|c|}{ (C) Tumor, individual cancers } \\
\hline Liver & 7 & 0.857 & 0.06 \\
\hline Breast & 5 & 0.800 & 0.19 \\
\hline Esophageal & 2 & I & 0.24 \\
\hline Head and neck & 2 & 1 & 0.25 \\
\hline Lung & 2 & I & 0.25 \\
\hline Cervical & 2 & I & 0.25 \\
\hline Gastric & 2 & 0.714 & 0.28 \\
\hline
\end{tabular}

The concordance and probability of error were calculated for the null-hypothesis that Osteopontin levels are not correlated with high risk for short survival. (A) All tumors combined in distinct types of clinical samples. (B) Plasma Osteopontin in individual cancers (for serum Osteopontin see main text). (C) Tumor Osteopontin in individual cancers. Bold values indicate $P<0.1$

diagonal in bar graphs (Figure 2B). To further quantify these ranked data, we determined the probability that for two patient groups, the one that had the higher Osteopontin rank would also have a higher grade or stage rank. In $66.3 \%$ of these comparisons, the group with higher Osteopontin rank was also the group with a higher tumor grade, which Monte Carlo analysis revealed to be significant $(P=0.004)$. Positive comparisons were also seen in $81.3 \%$ of cases for tumor stage $\mathrm{N}$ (node involvement, $P=0.01$ ), $54.5 \%$ of cases for tumor stage $\mathrm{T}$ (primary tumor, $P=0.28$ ), and $70 \%$ of cases with higher tumor stage $\mathrm{M}$ (metastasis, $P=0.18$ ).

For stage $\mathrm{T}$ and $\mathrm{M}$, the positive relationship identified in the comparison of ranks was not statistically significant, possibly due to insufficient sample size. Advantageously, the categorical analysis can be applied to heterogeneous data sets. By combining immunohistochemistry with the other published tests, we identified a statistically significant relationship between Osteopontin levels and all grade and stage measures, including $\mathrm{T}$ and $\mathrm{M}$, thus demonstrating the benefit of incorporating all of the available data within one analysis (Figure 2C). The categorical analysis had higher sensitivity than a conventional meta-analysis approach (Supplement 2).

In the early stages of transformation, tumor progression can be described as the transition from normal tissue to precancerous lesions (dysplasia, metaplasia), preinvasive cancer, and cancer. According to categorical meta-analysis, Osteopontin expression levels were significantly associated with the progression of eight cancers, independent in one, and inversely correlated in two (skin cancer and gestational trophoblastic tumor) (Table 5). Of note, while Osteopontin appears to be a cancer biomarker for 31 individual malignancies its levels were significantly reduced below normal in non-melanoma skin cancer and gestational trophoblastic tumor. This suggests a unique role for Osteopontin in these two malignancies.

\section{DISCUSSION}

High levels of Osteopontin in several cancers are indicative of a poor prognosis. Overall and disease-free survival are inversely

\begin{tabular}{|r|ccc|}
\hline A Clinical variable & Pearson $\chi^{2}$ & Studies & Patients \\
\hline Tumor grade & $P<0.001$ & 31 & 3370 \\
Tumor stage $-\mathrm{T}$ & $P<0.001$ & 20 & 1891 \\
Tumor stage $-\mathrm{N}$ & $P<0.001$ & 16 & 2069 \\
Tumor stage $-\mathrm{M}$ & $P<0.001$ & 10 & 961 \\
\hline
\end{tabular}

B Grade $(P=0.004)$
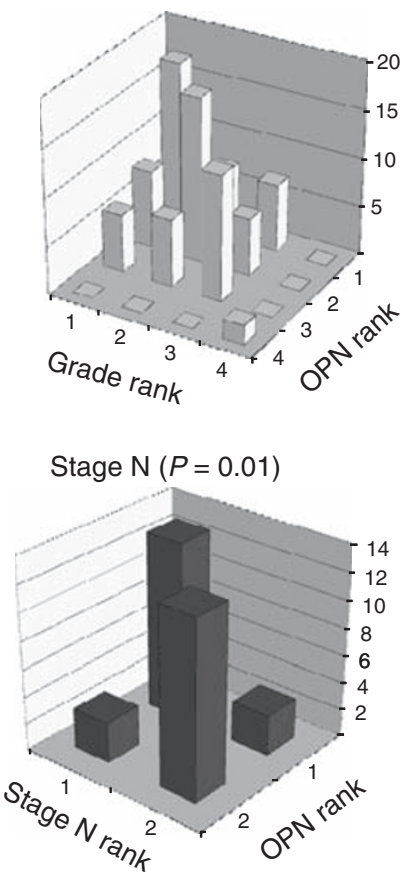

Stage $\mathrm{T}(P=0.28)$
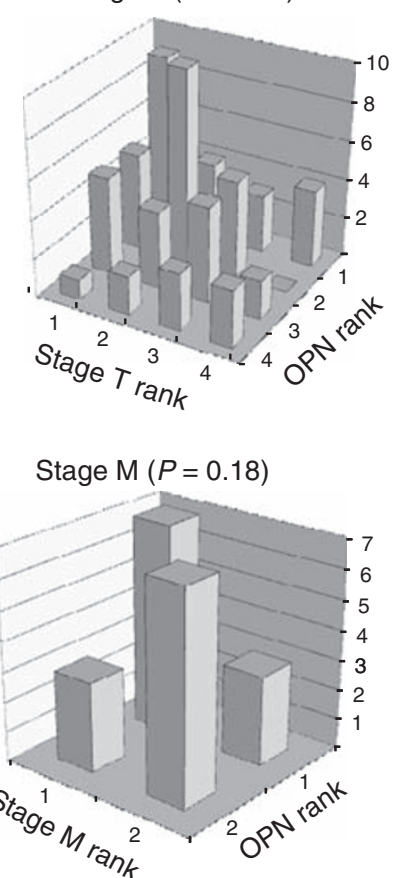

C

\begin{tabular}{|c|cc|}
\hline Clinical variable & Fraction & Significance \\
\hline Tumor grade & 0.67 & $P=0.002$ \\
Tumor stage $-\mathrm{T}$ & 0.7 & $P<0.001$ \\
Tumor stage $-\mathrm{N}$ & 0.84 & $P<0.001$ \\
Tumor stage $-\mathrm{M}$ & 0.82 & $P=0.006$ \\
\hline
\end{tabular}

Figure 2 By categorical meta-analysis, Osteopontin levels correlate with stage and grade of cancers. (A) The Pearson $\chi^{2}$ test of ranked Osteopontin immunohistochemistry scores with tumor grade and stage shows a significant dependence between Osteopontin rank and clinical variable. (B) The bar graphs of Osteopontin rank versus rank of grade or stage display an aggregation of data along the diagonal, indicating a positive relationship between Osteopontin levels and clinical variables. The associations are statistically significant for grade and node positivity, but not for stage $T$ and $M$. (C) Expanded analysis of grade and stage ranks to all published measures. In five studies with duplicate data sets only the immunohistochemistry results were used. We computed a measure analogous to that represented by the area under a ROC curve (see Methods). For all grade and stage measures, Osteopontin is a significant positive indicator.

related to Osteopontin levels in several cancers. There is strong correspondence between high Osteopontin and lower mean survival time in tumor (82\%) and plasma (100\%) measurements, with large mean differences in survival times, indicating a useful role for Osteopontin in patient stratification, Patient survival is largely determined by tumor aggressiveness. Hence, it is not unexpected that Osteopontin, a prognostic measure for survival, is also a marker for grade, stage, and early progression. It is likely 
Table 4 Categorical meta-analysis of tumor grade and stage

\begin{tabular}{|c|c|c|c|c|c|c|}
\hline Cancer type & Studies & Patients & Pearson P-value & Linear P-value & Correlation coefficient & Non-linear $P$-value \\
\hline All & 42 & 4408 & $<0.001$ & $<0.001$ & 0.27 & $<0.001$ \\
\hline Breast & 6 & 1061 & $<0.001$ & $<0.001$ & 0.28 & $<0.001$ \\
\hline Esophageal & 2 & 161 & $<0.001$ & 0.001 & -0.26 & $<0.001$ \\
\hline Gastric & 3 & 428 & $<0.001$ & $<0.001$ & -0.65 & $<0.001$ \\
\hline Glioma & 5 & 180 & $<0.001$ & $<0.001$ & 0.89 & $<0.001$ \\
\hline Lung & 4 & 610 & $<0.001$ & $<0.001$ & -0.24 & $<0.001$ \\
\hline Oral & 3 & 103 & $<0.001$ & 0.170 & 0.14 & $<0.001$ \\
\hline Ovarian & 5 & 379 & $<0.001$ & $<0.001$ & 0.68 & $<0.001$ \\
\hline Prostate & 2 & 117 & $<0.001$ & $<0.001$ & 0.45 & $<0.001$ \\
\hline Renal & I & 171 & $<0.001$ & $<0.001$ & I & I \\
\hline \multicolumn{7}{|c|}{ (B) Tumor stage (T) } \\
\hline Esophageal & 6 & 284 & $<0.001$ & $<0.001$ & 0.67 & $<0.001$ \\
\hline Gastric & 8 & 772 & $<0.001$ & $<0.001$ & 0.85 & $<0.001$ \\
\hline Head \& neck & 5 & 569 & $<0.001$ & $<0.001$ & 1.00 & N/A \\
\hline Liver & 4 & 497 & $<0.001$ & $<0.001$ & 0.89 & $<0.001$ \\
\hline Lung & 5 & 692 & $<0.001$ & $<0.001$ & 0.96 & $<0.001$ \\
\hline Myeloma & 1 & 30 & $<0.001$ & $<0.001$ & 1.00 & N/A \\
\hline Oral & 1 & 26 & $<0.001$ & $<0.001$ & -1.00 & 1 \\
\hline Ovarian & 8 & 444 & $<0.001$ & $<0.001$ & 0.22 & $<0.001$ \\
\hline Prostate & 1 & 47 & $<0.001$ & $<0.001$ & -1.00 & I \\
\hline Renal & I & 171 & $<0.001$ & $<0.001$ & 1.00 & N/A \\
\hline \multicolumn{7}{|c|}{ (C) Tumor stage (N) } \\
\hline All & 27 & 3159 & $<0.001$ & $<0.001$ & 0.81 & $<0.001$ \\
\hline Breast & 7 & 909 & $<0.001$ & $<0.001$ & 0.59 & N/A \\
\hline \multicolumn{7}{|c|}{ (D) Tumor stage (M) } \\
\hline All & 28 & 1900 & $<0.001$ & $<0.001$ & 0.72 & N/A \\
\hline Bladder & 1 & 23 & $<0.001$ & $<0.001$ & 1.00 & N/A \\
\hline Breast & 3 & 102 & $<0.001$ & $<0.001$ & 0.34 & N/A \\
\hline Colorectal & । & 10 & 0.002 & 0.003 & 1.00 & N/A \\
\hline Gastric & 4 & 612 & $<0.001$ & $<0.001$ & 1.00 & N/A \\
\hline Head \& neck & 3 & 113 & $<0.001$ & $<0.001$ & 1.00 & N/A \\
\hline Liver & 5 & 187 & $<0.001$ & $<0.001$ & -0.40 & N/A \\
\hline Lung & 6 & 644 & $<0.001$ & $<0.001$ & 0.75 & N/A \\
\hline Melanoma & 2 & 43 & $<0.001$ & $<0.001$ & 1.00 & N/A \\
\hline Oral & I & 26 & $<0.001$ & $<0.001$ & 1.00 & $\mathrm{~N} / \mathrm{A}$ \\
\hline Prostate & I & 10 & 0.002 & 0.003 & 1.00 & N/A \\
\hline Thyroid & i & 130 & $<0.001$ & $<0.001$ & 1.00 & N/A \\
\hline
\end{tabular}

Published Osteopontin levels in relation to tumor grade or tumor stage were analyzed. As a test for independence of the ranked data we used the Pearson $\chi^{2}$-test. To assess linear and non-linear trends of the ranked data we applied the Mantel-Haenszel $\chi^{2}$-test. N/A indicates that there were only two outcomes, and a non-linear fit is not measurable.

that patients with elevated Osteopontin at the time of diagnosis warrant more forceful treatment regimens than are suitable for patients with low levels of Osteopontin.

Although tumor grade, tumor stage, and early tumor progression are distinct measures for the clinical presentation of a cancer they are not mutually unrelated. A dedifferentiated, high grade tumor is more aggressive, and consequently more likely to disseminate and become high stage than a low grade tumor.
The molecular mechanisms driving progression, grade, and stage are overlapping. Osteopontin is associated with all of them. In patient care, the diagnosis and assessment of cancer is typically made on the basis of clinical and histo-morphologic criteria. However, molecular markers are more quantifiable and may be more reflective of underlying disease mechanisms. The incomplete convergence between clinical and molecular descriptors may require a reevaluation of how we assess cancer (Weber, 2010). 
Table 5 Categorical meta-analysis of tumor progression

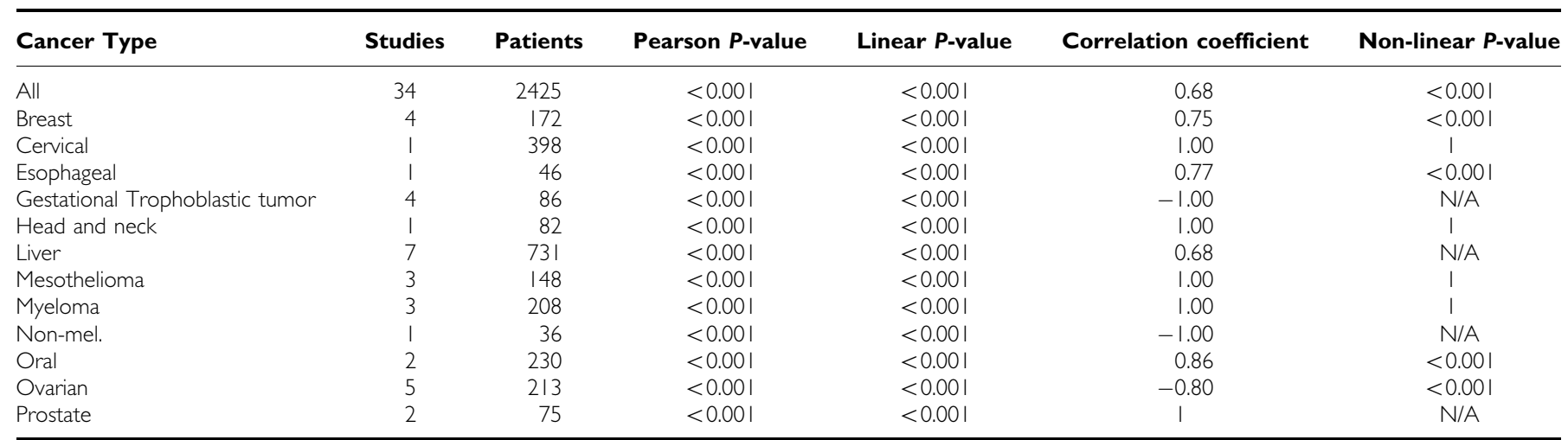

In the early stages of transformation, tumor progression can be described as the transition from normal tissue to precancerous lesions (dysplasia, metaplasia), preinvasive cancer, and cancer. The ranked levels of Osteopontin expression are significantly associated with the progression of liver cancer, myeloma, head and neck cancer, cervical cancer, prostate cancer, oral cancer, breast cancer, and mesothelioma. Unexpectedly, the meta-analysis reveals an inverse correlation to the progression of skin cancer and gestational trophoblastic tumor. Non-mel. = non-melanoma skin cancer.

In this analysis, the concordance between Osteopontin expression rank and stage or grade rank was $67-84 \%$ over all types of cancer. This is comparable to the accuracy commonly estimated for existing tumor markers, including CEA, CA 15-3, CA 19-9 and PSA (Ebert et al, 1996; Koopmann et al, 2006; Ulmert et al, 2009). When applied to select cancers, the accuracy of Osteopontin increases. Future research needs to assess whether the combination of Osteopontin with other markers can further improve its diagnostic value (Reinholz et al, 2002; O'Neill et al, 2005; Alonso et al, 2007; Ribeiro-Silva and Oliveira da Costa, 2008).

Meta-analysis has been a valuable tool in biomarker validation. One of its major limitations is the detection of true signals over the noise of heterogeneous input data. Categorical data analysis has a self-normalizing effect on study-to-study variations and may therefore be superior to conventional meta-regression algorithms.

\section{REFERENCES}

Agresti A (2007) 2nd edn. An Introduction to Categorical Data Analysis. John Wiley and Sons, Inc: Hoboken, NJ

Alonso SR, Tracey L, Ortiz P, Pérez-Gómez B, Palacios J, Pollán $M$ Linares J, Serrano S, Sáez-Castillo AI, Sánchez L, Pajares R, SánchezAguilera A, Artiga MJ, Piris MA, Rodríguez-Peralto JL (2007) A highthroughput study in melanoma identifies epithelial-mesenchymal transition as a major determinant of metastasis. Cancer Res 67: $3450-3460$

Ebert W, Muley T, Drings P (1996) Does the assessment of serum markers in patients with lung cancer aid in the clinical decision making process? Anticancer Res 16: $2161-2168$

Hong F, Breitling R (2008) A comparison of meta-analysis methods for detecting differentially expressed genes in microarray experiments. Bioinformatics 24: $374-382$

Hong F, Breitling R, McEntee CW, Wittner BS, Nemhauser JL, Chory J (2006) RankProd: a bioconductor package for detecting differentially expressed genes in meta-analysis. Bioinformatics 22: 2825-2827

Koopmann J, Rosenzweig CN, Zhang Z, Canto MI, Brown DA, Hunter M, Yeo C, Chan DW, Breit SN, Goggins M (2006) Serum markers in patients with resectable pancreatic adenocarcinoma: macrophage inhibitory cytokine 1 versus CA19-9. Clin Cancer Res 12: $442-446$
For the evaluation of Osteopontin as a biomarker for cancer, we have found conventional and categorical meta-analysis to be in agreement. This was not the case for the correlation of Osteopontin levels with tumor grade and stage (Figure 2 and Supplementary Figure S1). Here, the improved sensitivity of the categorical analysis is required to detect the existing trends in the published data sets.

\section{ACKNOWLEDGEMENTS}

This study was supported by NIH grant R43CA136011 to GFW.

Supplementary Information accompanies the paper on British Journal of Cancer website (http://www.nature.com/bjc)

Moher D, Liberati A, Tetzlaff J, Altman DG (2009) The PRISMA Group. Preferred reporting items for systematic reviews and meta-analyses: the PRISMA statement. BMJ 339: b2535

O'Neill CJ, Deavers MT, Malpica A, Foster H, McCluggage WG (2005) An immunohistochemical comparison between low-grade and high-grade ovarian serous carcinomas: significantly higher expression of p53, MIB1, BCL2, HER2/neu, and C-KIT in high-grade neoplasms. Am J Surg Pathol 29: 1034-1041

Reinholz MM, Iturria SJ, Ingle JN, Roche PC (2002) Differential gene expression of TGF-beta family members and osteopontin in breast tumor tissue: analysis by real-time quantitative PCR. Breast Cancer Res Treat 74: 255-269

Ribeiro-Silva A, Oliveira da Costa JP (2008) Osteopontin expression according to molecular profile of invasive breast cancer: a clinicopathological and immunohistochemical study. Int J Biol Markers 23: 154-160

Rice ME, Harris GT (2005) Comparing effect sizes in follow-up studies: ROC Area, Cohen's d, and r. Law Hum Behav 29: 615-620

Ulmert D, O'Brien MF, Bjartell AS, Lilja H (2009) Prostate kallikrein markers in diagnosis, risk stratification and prognosis. Nat Rev Urol 6: 384-391

Weber GF (2008) Molecular mechanisms of metastasis. Cancer Letters 270: $181-190$

Weber GF (2010) Toward a molecular classification of cancer. Toxicology (in press) 